\title{
Микроволновое излучение вспышечной петли при наличии турбулентности вистлеров
}

\author{
В.Ф. Мельников ${ }^{1}$, Л.В. Филатов ${ }^{2}$ \\ 1 Главная (Пулковская) астрономическая обсерватория РАН, Пулковское шоссе, 65/1, \\ Санкт-Петербург, 196140 \\ v.melnikov@gao.spb.ru \\ ${ }^{2}$ Нижегородский архитектурно-строительный университет, Ильинсая, 65, Нижний Новгород, 930950 \\ filatovlv@yandex.ru
}

Поступила в редакцию 15 ноября 2017 г.

Аннотация. Рассмотрено влияние вистлеров на распределение и гиросинхротронное излучение быстрых электронов, инжектированных в вершину корональной магнитной ловушки. Для неоднородной петлеобразной ловушки решается кинетическое уравнение в приближении Фоккера - Планка, учитывающее рассеяние быстрых электронов на частицах фоновой плазмы и на вистлерах, заполняющих ловушку. Рассчитывается микроволновое излучение и проводится сравнение рассеяния нетепловых электронов на вистлерах и на частицах плазмы. Проводится сравнительный анализ зависимости параметров излучения от типа инжекции быстрых электронов в петлю (изотропно и продольно). Показано, что при плотности энергии вистлеров выше определенной характеристики излучения петли значительно изменяются.

MICROWAVE RADIATION IN A FLARE LOOP IN THE PRESENCE OF WHISTLER TURBULENCE, by V.F. Melnikov, L.V. Filatov. The influence of whistlers on the distribution and gyrosynchrotron emission by fast electrons injected into a coronal magnetic trap is considered. For an inhomogeneous loop-like magnetic trap we solve the kinetic Fokker-Planck equation taking into account the scattering of fast electrons on the background plasma and whistler turbulance in it. Gyrosynchrotron microwave emission is calculated and the comparison of the influence of nonthermal electron scattering on whistlers and plasma is carried out. Also, a comparative analysis of the dependence of emission parameters on the two injection types (isotropic and longitudinal) is fulfilled. It is shown that the loop microwave emission characteristics change considerably under whistler energy density larger than some critical value.

Ключевые слова: Солнце, корона, вспышечные петли, гиросинхротронное излучение

\section{1 Введение}

Гиросинхротронное (ГС) микроволновое излучение нетепловых электронов во вспышечных петлях очень чувствительно к параметрам распределений электронов по энергии и питч-углам (Флейшман, Мельников, 2003). Поэтому оно может служить инструментом для диагностики этих распределений, а также таких процессов, как ускорение, инжекция и рассеяние частиц в солнечных 
вспышках. Рассеяние нетепловых электронов на частицах фоновой плазмы и на волновой турбулентности (БМЗ-волны, вистлеры, ионный звук, ленгмюровские волны) может заметно изменить параметры распределений электронов в петле. Характер кулоновских потерь энергии и рассеяния электронов в конус потерь в неоднородной по полю петле и их влияние на характеристики ГС-излучения к настоящему времени довольно хорошо исследованы (Флетчер, Мартенс, 1998; Мельников и др., 2002; Мельников и др., 2006). Вместе с тем наши знания о влиянии на ГС-излучение рассеяния нетепловых электронов на вистлерах явно недостаточны.

Известно, что вистлеры относятся к той же дисперсионной ветви, что и быстрые магнитозвуковые волны, а их частоты находятся в области между ионной и электронной гирочастотой: $\omega_{i}<\omega<\omega_{e}$. Они представляют собой циркулярно поляризованную волну. Волновая турбулентность вистлеров во вспышечной петле может существенно влиять на энергетический спектр и питчугловое распределение электронов и, соответственно, на характеристики наблюдаемого излучения (Каплан, Цытович, 1973; Браун, Мелроуз, 1977; Беспалов и др., 1991; Гамилтон, Петросян, 1992; Миллер, 1997; Степанов, Цап, 2002; Степанов и др., 2007).

Отметим, что в большинстве работ расчеты влияния проведены в предположении однородной магнитной ловушки (пробкотрон) и либо мгновенной, либо стационарной инжекции нетепловых электронов. В работе (Филатов, Мельников, 2017) решена задача для неоднородной вспышечной петли, в вершину которой изотропно по питч-углам и нестационарно во времени инжектируются нетепловые электроны. Целью настоящей работы является: 1) изучение влияния турбулентности вистлеров на распределения электронов и на характеристики ГС-излучения для случая анизотропной (вдоль петли) инжекции электронов; 2) сравнительный анализ эффектов рассеяния на вистлерах при различных типах нестационарной инжекции (изотропной и анизотропной).

\section{2 Описание модели}

Рассматривается одномерная модель вспышечной петли в виде симметричной магнитной трубки с неоднородным по длине магнитным полем и неоднородной плотностью фоновой плазмы. В вершине (центре) петли производится нестационарная инжекция нетепловых электронов. Быстрый электрон в каждый момент $t$ описывается дрейфовой координатой $s$ положения в петле, энергией $E$ (в долях $\left.m_{e} c^{2}\right)$, питч-углом $\alpha$ между вектором скорости электрона и продольным магнитным полем $B$, при этом $\mu=\cos (\alpha)$.

Модели для стационарного продольного магнитного поля $B(s)$ и плотности плазмы петли с длиной $2 l$ задаются в виде аппроксимирующих функций:

$$
\begin{gathered}
B(s)=B_{\text {min }}+\left(B_{\max }-B_{\text {min }}\right)(s / l)^{2}, \\
n(s)=n_{\min }+\left(n_{\max }-n_{\min }\right)(s / l)^{4}, \\
-l<s<+l .
\end{gathered}
$$

Инжекция моделируется функцией источника со степенным энергетическим спектром и гауссовым профилем по остальным фазовым координатам:

$$
\begin{gathered}
F(E, \mu, s, t)=F_{0}\left(E / E_{\text {min }}\right)^{-\delta} e^{-\left(\left(s-s_{0}\right) / s_{1}\right)^{2}}\left(e^{-\left(\left(\mu-\mu_{01}\right) / d\right)^{2}}+e^{-\left(\left(\mu-\mu_{02}\right) / d\right)^{2}}\right) e^{-\left(\left(t-t_{0}\right) / t_{1}\right)^{2}} \\
E_{\text {min }} \leq E \leq E_{\text {max }}
\end{gathered}
$$

Предполагается, что в петле распространяются электромагнитные волны свистового диапазона - вистлеры, источником которых является процесс конверсии быстрых магнитозвуковых (БМЗ) волн, образующихся непрерывно в области нестационарного вспышечного энерговыделения (Каплан, Цытович, 1973; Миллер, 1997). Плотность плазмы внутри вспышечных петель обычно выше, чем снаружи, поэтому для вистлеров вспышечная петля является волноводом, в котором они распространяются продольно. Возможное влияние на вистлеры быстрых электронов и других плазменных 
волн не учитывается. Полагаем, что плотность энергии $W(k, s)$ задана и постоянна во времени, а зависимость от волнового числа $k$ выражается степенным законом аналогично спектру Колмогорова (Гамилтон, Петросян, 1992; Миношима и др., 2011; Филатов, Мельников, 2017):

$$
\begin{gathered}
W(k, s)=\left[(q-1) / k_{\text {min }}\right]\left(k / k_{\text {min }}\right)^{-q} W_{t o t}, \\
W_{t o t}=\int_{k_{\text {min }}}^{\infty} W(k) d k=R B^{2}(s) / 8 \pi
\end{gathered}
$$

Здесь параметр $R$ показывает долю плотности энергии вистлеров по отношению к плотности магнитной энергии. Параметр $q$ показывает степень наклона спектра турбулентности вистлеров; $k_{m i n}-$ нижнее пороговое значение волнового вектора вистлеров.

При построении кинетической модели для быстрых электронов пренебрегаем кривизной петли и поперечными дрейфами в ней. Полагаем также, что процессы в магнитной ловушке адиабатичны, а частота столкновений электронов с частицами фоновой плазмы мала по сравнению с их гирочастотой. Для солнечных вспышечных петель эти предположения оправданы и тогда кинетическое уравнение для функции распределения быстрых электронов $f(E, \mu, s, t)$ будем использовать в квазилинейном диффузионном приближении Фоккера - Планка (Гамилтон и др., 1990). Коэффициент питч-угловой диффузии (рассеяния) в нем определяется как столкновениями с частицами плазмы, так и взаимодействием с вистлерами (Филатов, Мельников, 2017). Столкновительная часть диффузии определяется дальним кулоновским взаимодействием, а взаимодействие электронов с вистлерами определяется резонансным взаимодействием, причем учитывается только прямое влияние вистлеров на быстрые электроны.

Распределения электронов, полученные при численном решении уравнения Фоккера-Планка, далее используются для нахождения характеристик ГС-радиоизлучения. Излучательная способность и коэффициент поглощения ГС-излучения рассчитываются на основе точных выражений (Флейшман, Мельников, 2003). В данной работе мы ограничились рассмотрением свойств ГСизлучения в режиме оптически тонкого источника. В итоге нами получены частотные спектры по $(\omega)$, пространственные распределения и временные профили рассчитанных характеристик интенсивности микроволнового излучения магнитной петли $I(\omega, s, t)$.

\section{3 Результаты численных экспериментов}

Результаты проведенных численных экспериментов для функций $f(E, \mu, s, t)$ и $I(\omega, s, t)$ показаны на рис. 1-3. В численных расчетах исследовалось и сравнивалось влияние турбулентности вистлеров при различных значениях спектральной мощности турбулентности и различных режимах инжекции нетепловых электронов во вспышечную петлю. Параметры турбулентности вистлеров $q=1.7$ и $R=10^{-7}, 10^{-8}$ выбраны так, чтобы характерное время рассеяния $\tau$, в рассматриваемом диапазоне энергий электронов, соответствовало режиму слабой диффузии $\tau>\left(B_{\max } / B_{\min }\right) 2 l / \nu$ (Беспалов и др., 1991). Расчетные параметры магнитного поля $B(s)$ в петле были приняты следующими: $B_{\max } / B_{\min }=600$ Гс $/ 300$ Гс, а плотность фоновой плазмы $n_{\max } / n_{\min }=5 \cdot 10^{9} / 5 \cdot 10^{12} \mathrm{~cm}^{-3}$. Для выбранной расчетной сетки вершина петли $(L T)$ соответствует координате $s=7.5 \cdot 10^{7} \mathrm{~cm}$, $\left(\omega_{p} / \omega_{e}=0.42\right)$. Срединные области петли $(L G 1)$ и $(L G 2)$ соответствуют координатам $s=1 \cdot 10^{9} \mathrm{~cm}$ $\left(\omega_{p} / \omega_{e}=0.51\right)$ и $s=2 \cdot 10^{9} \mathrm{~cm},\left(\omega_{p} / \omega_{e}=0.37\right)$. Область вблизи основания петли $(F P)$ соответствует координате $s=2.5 \cdot 10^{9} \mathrm{~cm},\left(\omega_{p} / \omega_{e}=0.15\right)$, при полудлине всей петли $l=2.95 \cdot 10^{9}$ см.

Инжекция нетепловых электронов происходит в вершине $L T$ с полушириной $s_{1}=3 \cdot 10^{8}$ см, причем анизотропно по питч-углам, вдоль магнитного поля петли, с параметрами $\mu_{01}=1, \mu_{02}=-1, d=0.2$. Для сравнения, для тех же прочих условий мы рассматриваем случай изотропной инжекции: $d=\infty$. Максимум инжекции приходится на момент времени $t_{0}=2.5 \mathrm{c}$, а ее полудлительность $t_{1}=1.4 \mathrm{c}$ при общем времени модельного расчета $30 \mathrm{c}$. Нормировочная константа $F_{0}=10^{12} \mathrm{c}^{-1} \cdot \mathrm{cm}^{-3}$ задает поток быстрых электронов, инжектированных за единицу времени в единицу объема петли. Диапазон энергии электронов $E_{\min }=30$ кэВ, а $E_{\max }=10 \mathrm{MэВ.}$ 

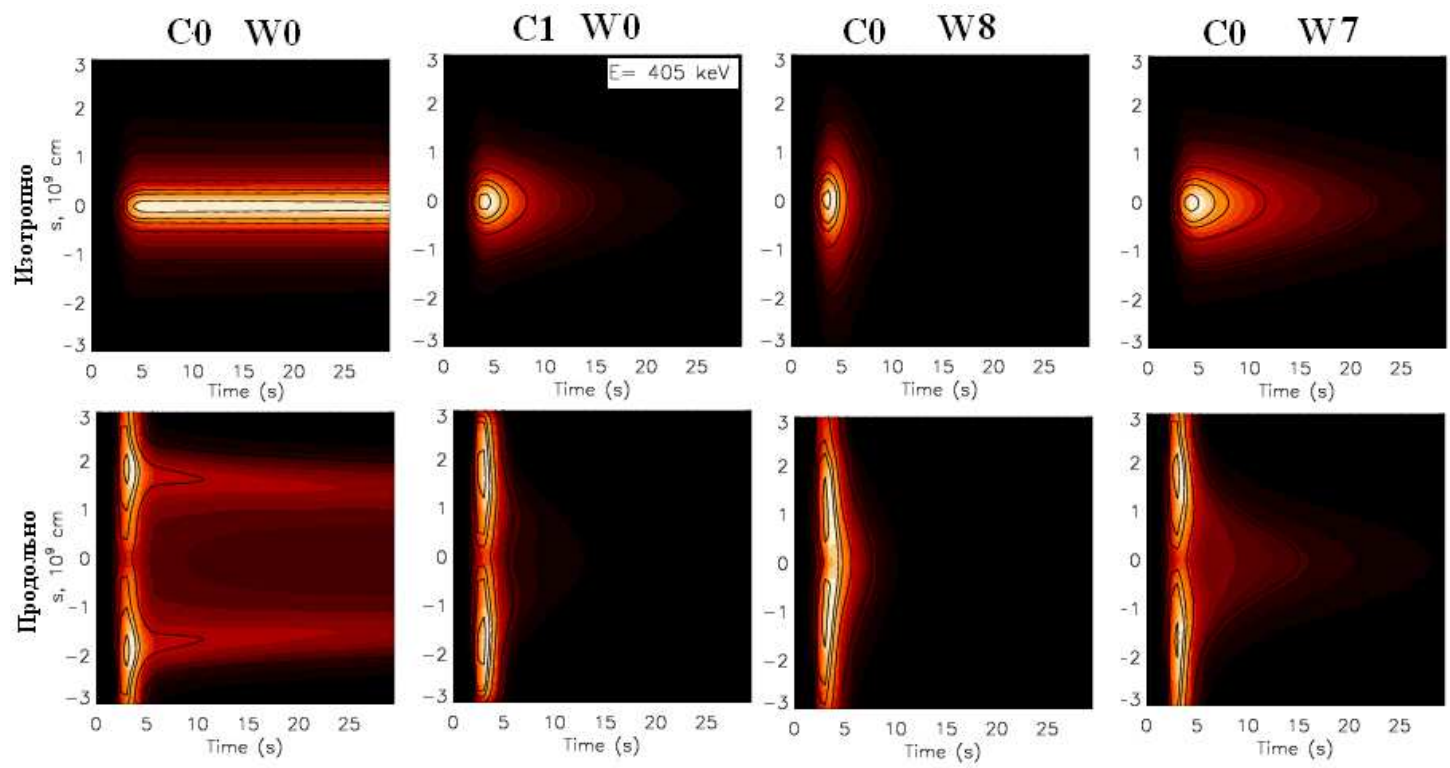

Рис. 1.

На рис. 1 приводятся распределения концентрации быстрых электронов с энергией $E=405$ кэВ на плоскости время - положение $(t-s)$ в яркостном представлении линий уровня. Верхние панели соответствуют изотропной инжекции, а нижние - анизотропной (продольной) инжекции. Крайние левые панели показывают распределения электронной концентрации для случая отсутствия взаимодействия инжектированных электронов как с частицами $(C 0)$, так и с вистлерами $(W 0)$. Панели $(C 1),(W 0)$ соответствуют режиму кулоновских столкновений и без учета взаимодействия с вистлерами. Предпоследние $(C 0 W 8)$ и последние $(C 0 W 7)$ панели справа иллюстрируют взаимодействие электронов только с турбулентностью с разной плотностью энергии вистлеров: $\mathrm{R}=10^{-8}$ и $\mathrm{R}=10^{-7}$ соответственно.

Из рис. 1 можно видеть значительные отличия в распределениях электронов вдоль ловушки для случаев анизотропной и изотропной инжекции. Для изотропной инжекции пик распределения находится в центре петли в течение всего времени инжекции. При продольной инжекции, наоборот, в центре петли наблюдается минимум, а вблизи оснований - максимумы. Однако этот тип распределения сохраняется только для случаев $(C 0 W 0)$ и $(C 1 W 0)$. При учете взаимодействия с вистлерами распределение нетепловых электронов вдоль петли изменяется: на фазе спада инжекции в центре петли появляется дополнительный пик. Особенно это заметно при большой плотности вистлеров $(C 0 W 7)$. Причина накопления нетепловых электронов в центре петли очевидно связана с фактом повышенного рассеяния по питч-углам на вистлерах.

На рис. 2 показаны временные профили интенсивности гиросинхротронного излучения на частотах 17 ГГц и 34 ГГц в вершине петли. Интенсивность излучения при продольной инжекции намного ниже из-за того, что большая часть инжектированных электронов высыпается в конус потерь и выходит из ловушки. Интересной особенностью случая с продольной инжекцией является то, что задержки пиков излучения относительно пика инжекции здесь более заметны. Особенно это проявляется в случае учета только кулоновских столкновений $(C 0 W 0)$, а также при малых плотностях энергии турбулентности вистлеров $(C 0 W 8)$. В целом учет вистлеров приводит к уменьшению задержек и более быстрому спаду интенсивности ГС-излучения для обоих типов инжекции. Этого и следовало ожидать, так как вистлеры эффективно рассеивают излучающие электроны в конус потерь. 

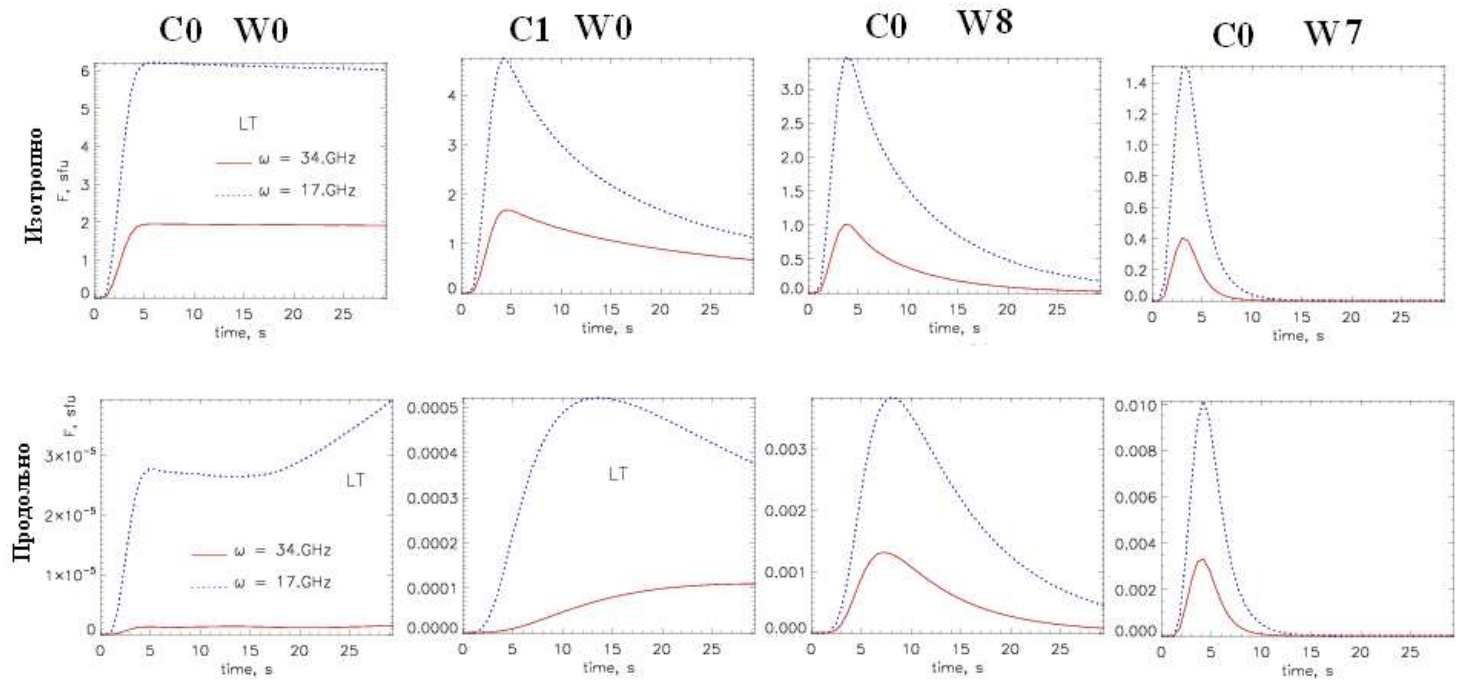

Рис. 2.
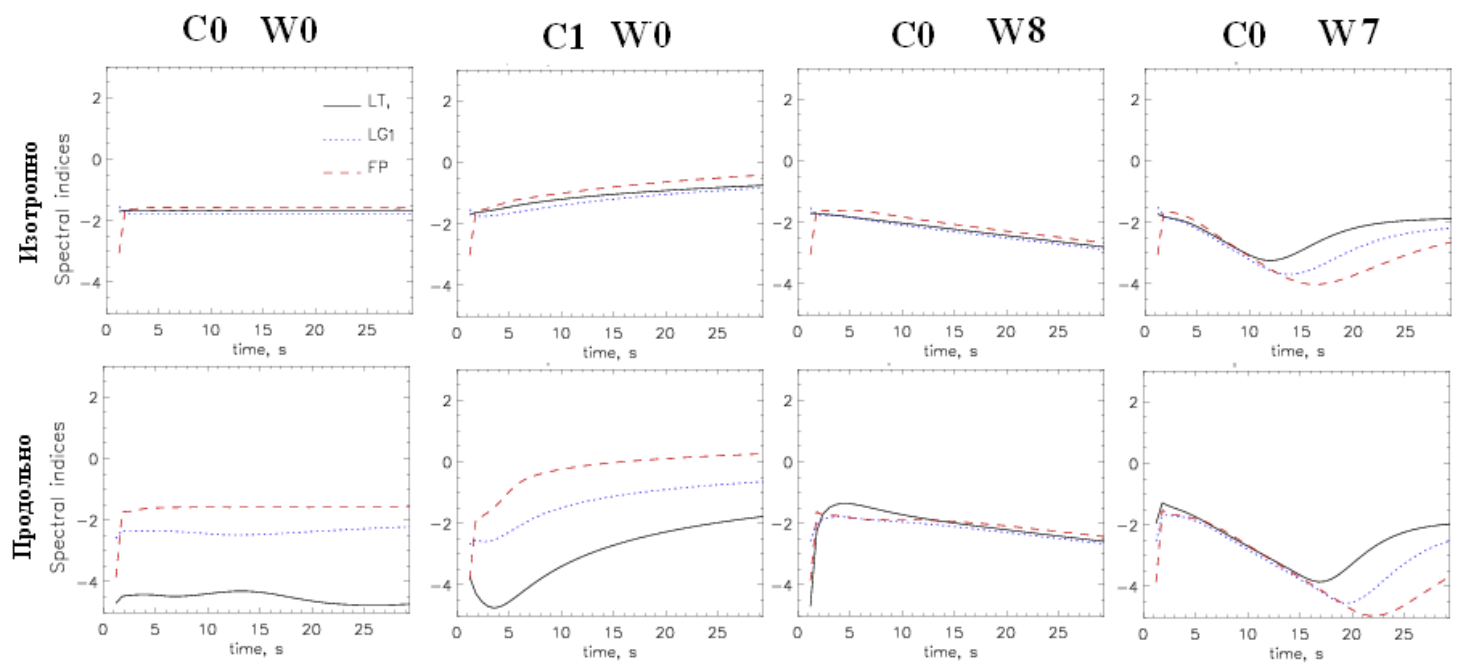

Рис. 3.

Рис. 3 показывает временную динамику спектрального индекса (наклона частотного спектра между частотами 17 ГГц и 34 ГГц). Видно, что качественно динамика спектрального индекса для анизотропной и изотропной инжекций отличается мало. Наклон частотного спектра монотонно уменьшается в случае учета только кулоновских столкновений $(C 1 W 0)$ и, наоборот, увеличивается в случае учета взаимодействия с вистлерами $(C 0 \mathrm{~W} 8, C 0 \mathrm{~W} 7)$. Очевидно, что для обоих типов инжекции причиной укручения частотного спектра является укручение энергетического спектра нетепловых электронов, связанное с фактом более эффективного резонансного взаимодействия с вистлерами электронов более высоких энергий. 


\section{4 Выводы}

Рассмотрено влияние вистлеров на распределение и гиросинхротронное излучение быстрых электронов, нестационарно инжектированных в вершину неоднородной магнитной ловушки. Для расчета распределений электронов в ловушке решается кинетическое уравнение в приближении ФоккераПланка, учитывающее рассеяние быстрых электронов на частицах фоновой плазмы и на вистлерах, заполняющих ловушку. Рассчитывается микроволновое излучение от полученных распределений электронов и проводится сравнение влияния рассеяния нетепловых электронов на вистлерах и на частицах плазмы, а также сравнение влияния типа инжекции в магнитную петлю (изотропно и анизотропно). Показано, что при плотности энергии вистлеров выше определенной характеристики излучения петли значительно изменяются как для случая изотропной, так и для случая анизотропной инжекции. Главное отличие случаев с анизотропной и изотропной инжекциями состоит в том, что для изотропной инжекции имеется единственный пик распределения концентрации электронов и интенсивности ГС-излучения вдоль петли в вершине (центре) петли, тогда как для анизотропной имеют место два пика вблизи оснований петли. В случае анизотропной инжекции учет рассеяния электронов на вистлерах приводит к сглаживанию провала между пиками вблизи оснований. Влияние вистлеров на частотный спектр ГС-излучения проявляется прежде всего в укручении спектра в оптически тонкой области. Учет только кулоновских столкновений приводит к прямо противоположному эффекту: частотный спектр уплощается. Это характерно для обоих типов инжекции.

Работа выполнена при поддержке грантов РНФ 16-12-10448, РФФИ 17-52-80064, 18-02-00856.

\section{Литература}

Беспалов и др. (Bespalov P.A., Zaitsev V.V., Stepanov A.V.) // Astrophys. J. 1991. V. 374. P. 369.

Браун, Мелроуз (Brown J.C., Melrose D.B.) // Solar Phys. 1977. V. 52. № 4. P. 117.

Гамилтон и др. (Hamilton R.J., Lu E.T., Petrosian V.) // Astrophys. J. 1990. V. 354. P. 726.

Гамилтон, Петросян (Hamilton R.J., Petrosian V.) // Astrophys. J. 1992. V. 398. P. 350.

Каплан, Цытович (Kaplan S.A., Tsytovich V.N.) // Plasma astrophysics. Oxford: Pergamon Press. 1973.

Мельников В.Ф., Горбиков С.П., Резникова В.Э., Шибасаки К. // Известия РАН. Серия физическая. 2006. Т. 70. С. 1472.

Мельников и др. (Melnikov V.F., Shibasaki K., Reznikova V.E.) // Astrophys. J. 2002. V. 580. P. 185.

Миллер (Miller J.A.) // Astrophys. J. 1997. V. 491. P. 939.

Миношима и др. (Minoshima T., Masuda S., Miyoshi Y., Kusano K.) // Astrophys. J. 2011. Issue 2. id. 111.

Степанов, Цап (Stepanov A.V., Tsap Y.T) // Solar Phys. 2002. V. 211. P. 135.

Степанов и др. (Stepanov A.V., Yokoyama T., Shibasaki K., Melnikov V.F.) // Astron. Astrophys. 2007. V. 465. P. 613.

Филатов, Мельников (Filatov L.V., Melnikov V.F.) // Geomag. Aeron. 2017. V. 57. № 8. P. 1001.

Флейшман, Мельников (Fleishman G.D., Melnikov V.F.) // Astrophys. J. 2003. V. 587. № 2. P. 823.

Флетчер, Мартенс (Fletcher L., Martens P.C.H.) // Astrophys. J. 1998. V. 505. P. 418. 